\title{
The Impact of Turbulent Solar Wind Fluctuations on Solar Orbiter Plasma Proton Measurements
}

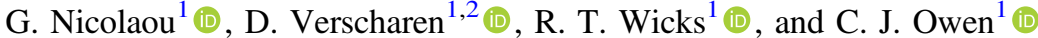 \\ ${ }^{1}$ Department of Space and Climate Physics, Mullard Space Science Laboratory, University College London, Dorking, Surrey, RH5 6NT, UK; g.nicolaou@ucl.ac.uk \\ ${ }^{2}$ Space Science Center, University of New Hampshire, NH, USA \\ Received 2019 June 18; revised 2019 September 24; accepted 2019 September 26; published 2019 November 25
}

\begin{abstract}
Solar Orbiter will observe the Sun and the inner heliosphere to study the connections between solar activity, coronal structure, and the origin of the solar wind. The plasma instruments on board Solar Orbiter will determine the three-dimensional velocity distribution functions of the plasma ions and electrons with high time resolution. The analysis of these distributions will determine the plasma bulk parameters, such as density, velocity, and temperature. This paper examines the effects of short-timescale plasma variations on particle measurements and the estimated bulk parameters of the plasma. For the purpose of this study, we simulate the expected observations of solar wind protons, taking into account the performance of the Proton-Alpha Sensor (PAS) on board Solar Orbiter. We particularly examine the effects of Alfvénic and slow-mode-like fluctuations, commonly observed in the solar wind on timescales of milliseconds to hours, on the observations. We do this by constructing distribution functions from modeled observations and calculate their statistical moments in order to derive plasma bulk parameters. The comparison between the derived parameters with the known input allows us to estimate the expected accuracy of Solar Orbiter proton measurements in the solar wind under typical conditions. We find that the plasma fluctuations due to these turbulence effects have only minor effects on future SWA-PAS observations.
\end{abstract}

Key words: plasmas - solar wind - turbulence - waves

\section{Introduction}

As the solar wind expands into the heliosphere, it develops a strong turbulent character (e.g., Tu \& Marsch 1995; Marsch 2006; Bruno \& Carbone 2013), with spatial and temporal variations over a wide range of scales (e.g., Goldstein et al. 1995; Verscharen et al. 2019). Numerous studies have revealed the nature of the turbulence at different scales, identifying Alfvénic fluctuations (Belcher \& Davis 1971), magnetoacoustic (fast and slow MHD) fluctuations, and pressure-balanced structures (Tu \& Marsch 1995; Bruno \& Carbone 2013) at large scales, and the contribution of fluctuations with polarization properties of kinetic Alfvén waves, slow modes, and whistler modes at small scales (Gary \& Smith 2009). The presence of these fluctuations makes the study of the plasma kinetic state at a given time challenging, as the plasma kinetic state constantly changes self-consistently in response to the turbulence fluctuations (e.g., Marsch 2006, \& references therein). Moreover, at small scales, the plasma and field fluctuations do not follow Gaussian statistics and exhibit properties of intermittency, increasing the complexity of the system (e.g., Matthaeus et al. 2015; Wan et al. 2016).

In situ plasma observations provide the information to study the kinetic properties and the dynamics of the solar wind. The three-dimensional (3D) velocity distribution function (VDF) of the plasma particles, at a given time, contains the information to derive the plasma bulk parameters, such as the density, velocity, and temperature. Past and future solar wind missions have been designed to study the solar wind by obtaining the 3D VDFs of its component populations with a time resolution ranging from a few seconds to more than 1 minute. However, the effect of the highly dynamic nature of the solar wind on the accuracy of the measurements has not been often considered.

For example, the Helios probes were launched in the mid 1970s and operated in a heliocentric orbit, reaching a perihelion of about 0.3 au to study the solar wind in the inner heliosphere for the first time. The plasma experiment E1 on board Helios was designed to measure the solar wind plasma particles and determine their 3D VDFs (Schwenn et al. 1975; Rosenbauer et al. 1977). In the experiment's nominal operation mode, Helios data provided the full 3D VDF of protons every $\sim 40 \mathrm{~s}$.

The Wind spacecraft was launched in 1994 and is dedicated to investigating basic plasma processes in near-Earth space. It has been in a halo orbit around $L_{1}$ since 2004. Wind's Solar Wind Experiment is a comprehensive plasma instrument, measuring the distributions of protons and heavier ions (Ogilvie et al. 1995). It carries a Faraday cup subsystem, which, in a nominal mode, provides the measurements to determine the densities, bulk velocities, and temperatures of solar wind ions every $92 \mathrm{~s}$. Wind's 3D plasma and energetic particle investigation instrument, Wind/3DP (Lin et al. 1995), carries a set of proton electrostatic analyzers and a set of electron electrostatic analyzers that measure the 3D VDFs of the corresponding species every $3 \mathrm{~s}$.

Solar Orbiter is scheduled for launch in 2020 February. It is designed to study the inner heliosphere, which in part it will do by measuring the solar wind plasma in situ with a higher time resolution than previous missions. The Solar Wind Analyser's Proton-Alpha Sensor (SWA-PAS) on board Solar Orbiter is an electrostatic analyzer that will measure the 3D VDF every $4 \mathrm{~s}$.

There are technological limitations that prevent simultaneous observations of the entire 3D VDF in infinitesimal time intervals. Typical plasma sensors, such as those mentioned above, scan through the energy and flow direction of the particles in discrete consecutive steps, measuring the particle flux at each step in a given time interval (acquisition time). As a result, within the measurement time for a full 3D VDF, the individual instrument samples are affected by any fluctuations of the distribution function that occur on shorter timescales. 
Such small-scale variations affect the observed VDF and thus the estimated plasma bulk parameters. For example, when a relatively sharp discontinuity passes over the spacecraft, while the instrument performs a 3D VDF scan, the bulk velocity may rapidly change. In such a case, the instrument may observe parts of two very different VDFs for each "half" of its scan. If the resulting observation is interpreted as them being one VDF, the results are distorted. Any later analysis of moments will be wrong, as they will neither correspond to the upstream nor the downstream plasma region, nor indeed any part of the boundary itself.

In an example, Verscharen \& Marsch (2011) showed that wave activity can lead to artificial temperature anisotropies in the observed plasma distributions. Large-amplitude waves can shift the VDF in the direction perpendicular to the background magnetic field. Because these fluctuations occur at timescales smaller than the instrument's sampling time, the observed average distribution exhibits a broadening in the perpendicular direction, which eventually could be misinterpreted as an intrinsic temperature anisotropy. In a more recent study, Nicolaou et al. (2015a) demonstrated that random variations in the plasma bulk parameters result in broader VDFs, which eventually lead to a bias toward higher temperatures. The authors considered observations of plasma ions in the distant Jovian tail by the Solar Wind Around Pluto Instrument (McComas et al. 2008) on board New Horizons.

In this paper, we predict the effects of temporal variations due to turbulence on measurements with Solar Orbiter's SWAPAS. We adopt the well-established forward-modeling technique by modeling the instrument response in a simulated plasma environment (see also Vaivads et al. 2016; Cara et al. 2017; Wilson et al. 2017; Kim et al. 2019). We specifically consider the characteristic solar wind plasma behavior due to Alfvénic and slow-mode-like waves turbulence. Early observations of the solar wind (e.g., Belcher \& Davis 1971) showed that proton velocity and magnetic-field fluctuations are highly correlated for a majority of the time. This is the characteristic signature of Alfvén waves; plasma waves with fluctuations transverse to the magnetic-field direction. Detailed data analyses over the past 40 years have shown that Alfvénic modes carry the majority of the energy in the free-flowing solar wind (e.g., Roberts 2010; Wicks et al. 2013). More recent statistical analyses have shown that there is a minor component of slow-mode waves (e.g., Klein et al. 2012; Verscharen et al. 2017), which are longitudinal compressive waves. These two wave fields act to distort the proton VDF measurement by fluctuating the plasma on the timescale over which the observation is made (Verscharen \& Marsch 2011).

In this study, we model the expected observations in such turbulent conditions, and quantify the error of the plasma parameters derived from the moments of the 3D VDF. Our study could be extended for the diagnosis of the errors of SWA-PAS plasma observations. In the following section, we describe SWA-PAS, and in Section 3, we describe the method we use to simulate the expected observations and our standard techniques to analyze them. In Section 4, we present our results, which we discuss in detail in Section 5. We also discuss and compare the expected errors in the measurements of previous missions. The model that we use for the solar wind turbulence is included in the Appendix.

\section{Instrumentation}

SWA consists of three sensors: (i) The Proton-Alpha Sensor (SWA-PAS), (ii) the Electron Analyser System (SWA-EAS), and (iii) the Heavy Ion Sensor (SWA-HIS). The three sensors share a common Data Processing Unit (DPU) and are designed to measure the 3D VDFs of the solar wind particles. We use an idealized model of SWA-PAS, which is designed to observe the energy-per-charge range from 0.2 to $20 \mathrm{keV} / \mathrm{q}$. We consider a specific operation mode in which this range is covered in 96 exponentially spaced steps with a resolution of $\Delta E / E \sim 7.5 \%$. The azimuth field of view (F.O.V.) ranges from $-24^{\circ}$ to $+42^{\circ}$ with respect to the Sun direction, accounting for the expected range of the aberration angle, and is covered by 11 sectors that consist of individual channel electron multipliers (CEMs). The elevation F.O.V. ranges from $-22.5^{\circ}$ to $+22.5^{\circ}$ with respect to the Sun direction and is covered by nine electrostatic steps performed by the electrostatic deflector system (see Figures 1(a) and (b)). In the operation mode we consider here, the instrument performs one full 3D scan by repeating 9 elevation scans for each of the 96 energy steps, while for each energy and elevation pair, the 11 CEMs record the azimuth directions simultaneously. The instrument scans energies from highest to lowest, while it scans the elevation angles from top to bottom and from bottom to top, in consecutive order (see Figure 1(c)). The acquisition time $(\Delta \tau)$ for each energy and elevation direction is $\sim 1 \mathrm{~ms}$. A full 3D VDF is obtained in $\sim 1 \mathrm{~s}$, followed by $\sim 3 \mathrm{~s}$ of no measurement, resulting in an overall $\sim 4$ s cadence. We develop a model of SWA-PAS based on its initial calibration and ideal response for simplicity. We also neglect the voltage transition time during the energy-elevation scans.

\section{Data and Instrument Simulation}

SWA-PAS will measure the plasma at heliospheric distances between $\sim 0.3$ and $\sim 1$ au. Within this range, the average density $n_{0}$ is expected to be between $\sim 1$ and $\sim 50 \mathrm{~cm}^{-3}$, the average temperature $T_{0}$ is expected to be between a few eVs and $\sim 50 \mathrm{eV}$, the average magnetic field $B_{0}$ is expected to be between $\sim 1$ and $\sim 40 \mathrm{nT}$, and the average bulk speed is $\sim 500$ $\mathrm{km} \mathrm{s}^{-1}$ (e.g., Barouch 1977; Freeman 1988). For this paper, we model plasma turbulence for $n_{0}=20 \mathrm{~cm}^{-3}, T_{0}=20 \mathrm{eV}$, $B_{0}=10 \mathrm{nT}$, and $u_{0}=500 \mathrm{~km} \mathrm{~s}^{-1}$, which we consider typical values within the expected ranges. For these background plasma parameters and magnetic field, the Alfvén speed $V_{\mathrm{A}} \sim 50 \mathrm{~km} \mathrm{~s}^{-1}$, the plasma beta $\beta_{\mathrm{p}} \sim 1.6$, and the proton gyroradius $\rho_{\mathrm{g}} \sim 65 \mathrm{~km}$. We model the fluctuations of the plasma parameters considering Alfvénic and slow-mode-like turbulence. The Alfvénic component introduces fluctuations mainly in the velocity component perpendicular to the magnetic field. The slow-mode-like component is the minor component of the turbulent spectrum, but introduces density fluctuations in the frequency domain above the kinetic scales. For simplicity, we construct the turbulence spectrum considering that the waves are frozen in the plasma flow, which is known as Taylor's hypothesis (Taylor 1938). This simplification allows us to model a turbulent spectrum with power levels and polarization properties that match typical spacecraft observations. However, it is currently a matter of ongoing research to what degree Taylor's hypothesis is applicable to the types of fluctuations we discuss (e.g., Howes et al. 2014; Klein et al. 2014, 2015; Narita 2017; Perri et al. 2017; Bourouaine \& Perez 2018). We 


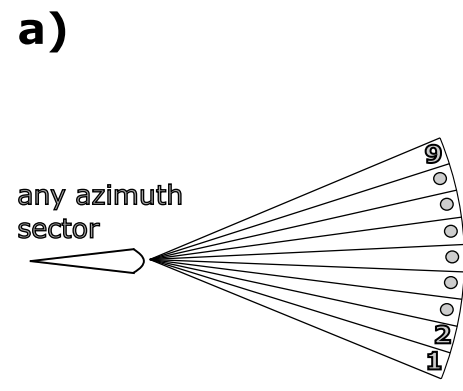

F.O.V. in elevation b)

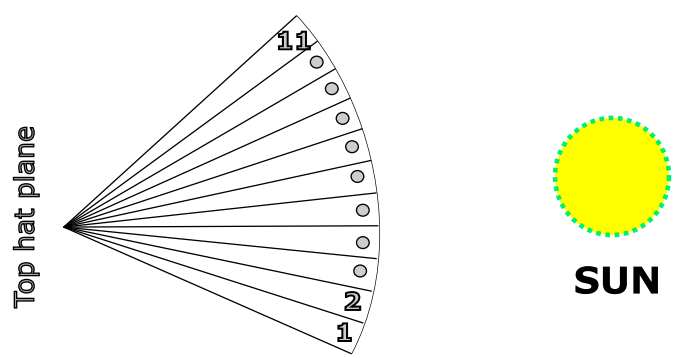

F.O.V. in azimuth

c)

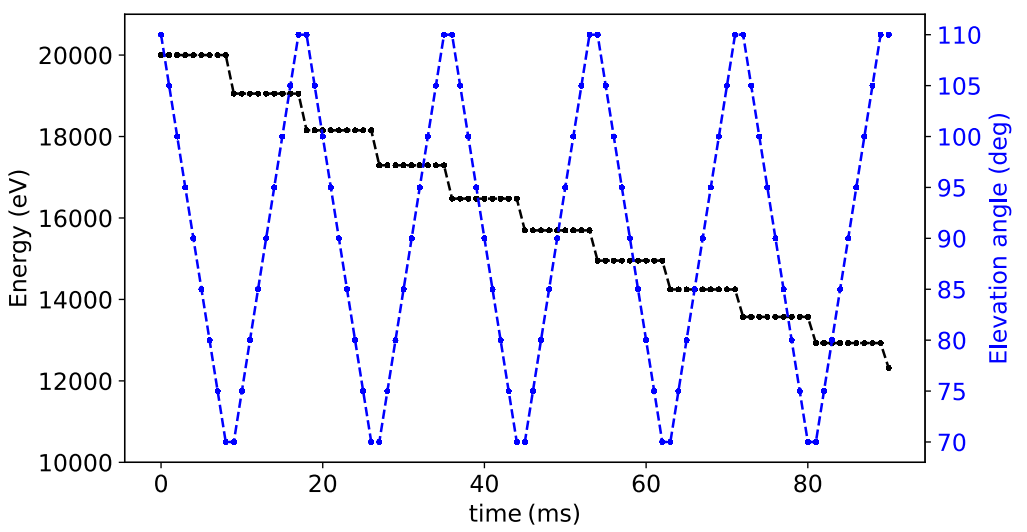

Figure 1. (a) The elevation and the (b) azimuth field of view of our SWA-PAS model. (c) An example of the instrument's energy $\times$ elevation scans during modeled operations. We show the first 10 energy $\times$ elevation scans, as the instrument scans the elevation directions from top to bottom and from bottom to top in consecutive order (blue line) for each energy step, starting from the highest energy (black line).

describe our calculation of the solar wind input distributions and their fluctuations due to turbulence in the Appendix. In the next subsections, we define our instrument model and the analysis of modeled measurements for specific 3D solar wind input VDFs, taking into account the SWA-PAS response.

\subsection{SWA-PAS Observation Model}

SWA-PAS measures the number of particles that enter the instrument aperture in each acquisition step $\tau$ at the specific energy $E(\tau)$, elevation $\Theta(\tau)$, and azimuth sector $\Phi$. The measured energy and elevation directions are functions of time, based on the sequential sampling process of the sensor (see Section 2). We calculate the expected counts $C(E(\tau), \Theta(\tau), \Phi, \tau)$ to be obtained at each acquisition step $\tau$ based on our modeled distribution $f$ as

$$
\begin{gathered}
C(E(\tau), \Theta(\tau), \Phi, \tau)=\frac{2}{m^{2}} \int_{E-\frac{\Delta E}{2}}^{E+\frac{\Delta E}{2}} \int_{\Theta-\frac{\Delta \Theta}{2}}^{\Theta+\frac{\Delta \Theta}{2}} \int_{\Phi-\frac{\Delta \Phi}{2}}^{\Phi+\frac{\Delta \Phi}{2}} \int_{\tau-\frac{\Delta \tau}{2}}^{\tau+\frac{\Delta \tau}{2}} \\
A_{\text {eff }}(\epsilon, \theta, \phi) f(\epsilon, \theta, \phi, t) \epsilon d \epsilon \cos \theta d \theta d \phi d t
\end{gathered}
$$

where $m$ is the mass of a measured particle and $A_{\text {eff }}$ is the effective area of the sensor. The 3D VDF $f$ is expressed in spherical coordinates, where $\epsilon$ is the particle energy, $\theta$ is the elevation angle, $\phi$ is the azimuth angle, and $t$ is the time. The energy resolution $\Delta E / E$ and the angular resolution in elevation and azimuth direction, $\Delta \Theta$ and $\Delta \Phi$ respectively, are considered constant for simplicity. As in Nicolaou et al. (2018), we assume that $A_{\text {eff }}$ is a discrete function of the elevation step $\Theta$ only, i.e., $A_{\text {eff }}(\epsilon, \theta, \phi) \equiv A_{\text {eff }}(\Theta)=A_{0} / \cos \Theta$. The independence of $A_{\text {eff }}$ on $\Phi$ assumes that the detection efficiency of the 11 CEMs in PAS is identical. Additionally, because we want to investigate specifically the effects of short- period turbulence fluctuations on the expected observations, we intentionally exclude statistical uncertainties (Poisson error) and any other physical source of statistical and systematical errors; such as background radiation, electronics noise, and contamination of the detectors. With these simplifications, we calculate the expected counts as

$$
\begin{gathered}
C(E(\tau), \Theta(\tau), \Phi, \tau)=\frac{2}{m^{2}} A_{0} E^{2} \frac{\Delta E}{E} \Delta \Theta \Delta \Phi \\
\times \sum_{\tau-\frac{1}{2} \Delta \tau}^{\tau+\frac{1}{2} \Delta \tau} f(\epsilon=E, \theta=\Theta, \phi=\Phi, t) d t,
\end{gathered}
$$

in which the integral over time in Equation (1) is solved numerically.

\subsection{Analysis of SWA-PAS Modeled Observations}

Most space-plasma analyses assume that $f$ remains constant during a full VDF scan period of the particle instrument. Under 

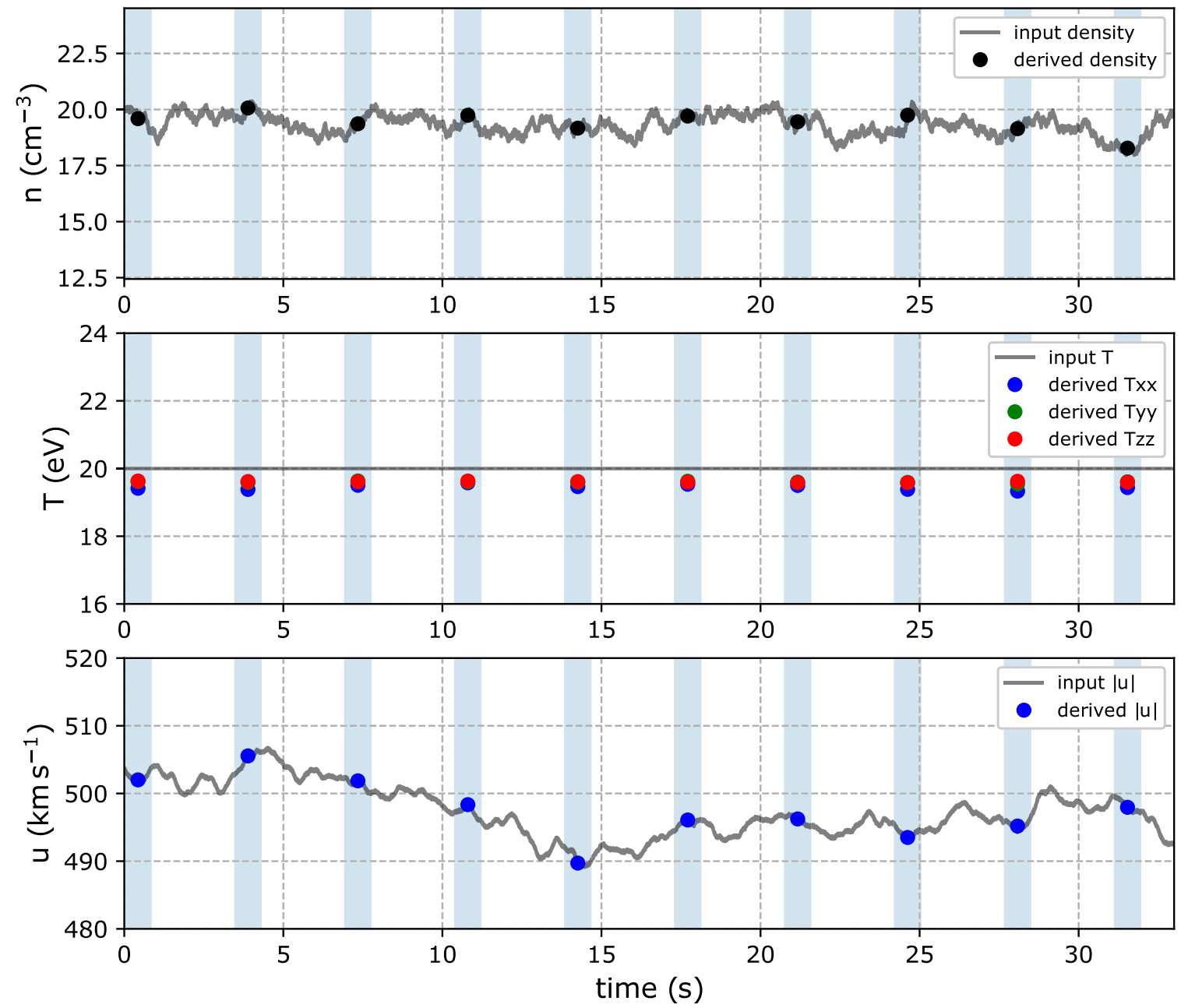

Figure 2. Time series of modeled solar wind with a turbulent spectrum consisting of Alfvén waves and slow modes and a comparison to derived moment parameters from the expected SWA-PAS observations at lower resolution. Each panel shows the input data (gray line) and the moments derived from the modeled observations (bullets). The shadowed areas represent the time intervals in which the instrument collects counts to construct an entire 3D VDF. The top panel shows the plasma density $\left(n=n_{0}+\Delta n\right)$, the middle panel shows the diagonal elements of the plasma temperature tensor $\left(T=T_{0}\right)$, and the bottom panel shows the plasma bulk speed $\left(u=\left|\boldsymbol{u}_{0}+\Delta \boldsymbol{u}\right|\right)$. Besides the small systematic underestimation of the plasma density and plasma temperature, the derived moments suggest that the accuracy of SWA-PAS measurements, under typical turbulent solar wind conditions, is remarkably high.

this assumption, Equation (1) becomes

$$
\begin{aligned}
C(E, \Theta, \Phi)= & \frac{2}{m^{2}} A_{0} E^{2} \frac{\Delta E}{E} \Delta \Theta \Delta \Phi \\
& \times f(\epsilon=E, \theta=\Theta, \phi=\Phi) \Delta \tau,
\end{aligned}
$$

which we invert to calculate the distribution function from the observed counts as

$$
f_{\text {out }}(E, \Theta, \Phi) \approx \frac{m^{2} C(E, \Theta, \Phi)}{2 E^{2} G}
$$

where

$$
G=A_{0} \frac{\Delta E}{E} \Delta \Theta \Delta \Phi \Delta \tau
$$

is the geometric factor of the instrument (for more details, see Nicolaou et al. 2018). The common application of Equation (4) in space-plasma analyses introduces inaccuracies if there are changes in $f$ at timescales shorter than the sampling time for a full 3D VDF.

In order to construct our modeled observations in a timevarying plasma, we take into account variations during the scanning sequences of the instrument. As the instrument scans in energy and elevation we vary $f$ using a model of Alfvénic and slow-mode-like turbulence, suitable for the solar wind (see the Appendix). The turbulent fluctuations cause $f$ to vary in time and so introducing inaccuracies in the determination of $f_{\text {out }}$ from the above assumption of time invariance, as discussed, in Equation (3). We then derive the distribution function from counts using Equation (4) under the discussed assumptions, and calculate its bulk parameters as moments. We compare the derived moments with those used to model the solar-wind plasma in the first place. This comparison allows us to quantify the error of the estimated plasma parameters due to underresolved variations of the plasma.

\section{Results}

Figure 2 shows the first $33 \mathrm{~s}$ of the modeled solar-wind proton bulk parameters for the input turbulence conditions described in the Appendix and the corresponding analysis of SWA-PAS modeled observations. The derived parameters are, by eye, in good agreement with the input parameters. In order to quantify the error of the estimated parameters due to the modeled turbulence, we construct histograms of density, 

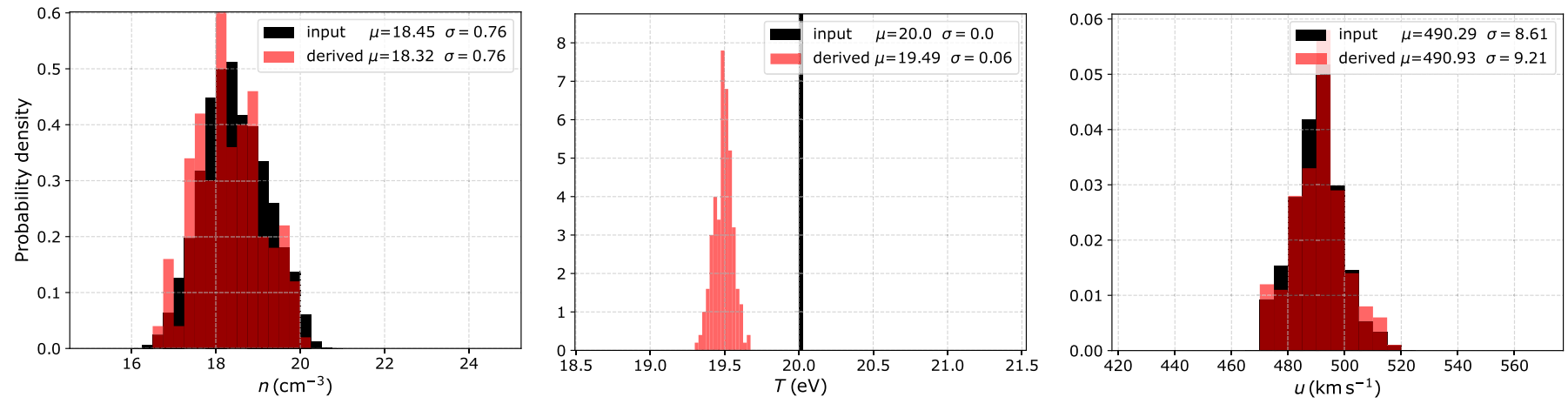

Figure 3. Histograms of the derived moments (red) and the average input moments (black) over the instrument's 3D VDF measurement intervals (black). The left panel shows the plasma density, the middle panel shows the scalar temperature, and the right panel shows the bulk speed. For this analysis, we analyze a sample of 200 modeled observations.
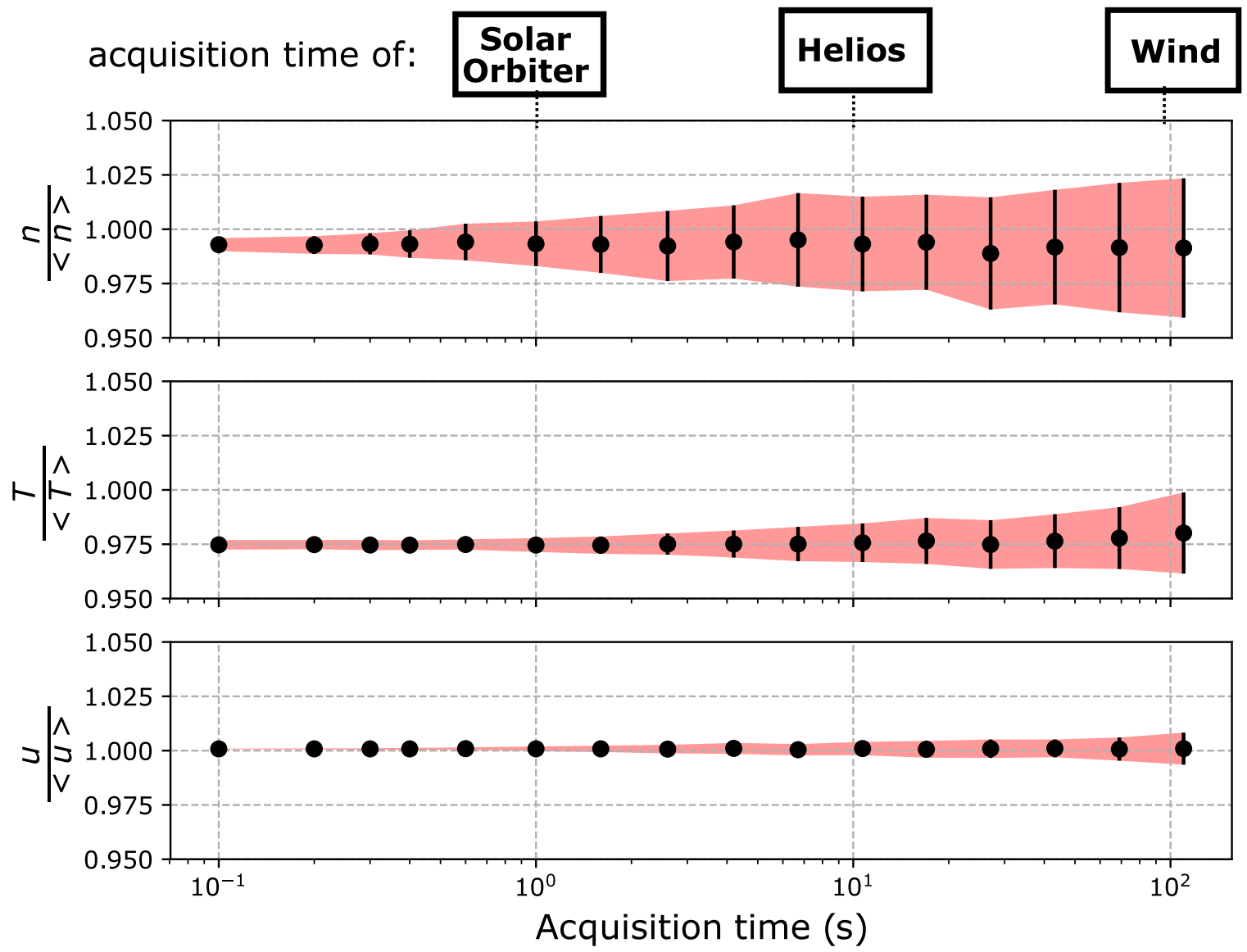

Figure 4. Average values and standard deviations of the normalized derived density (top), scalar temperature (middle) and bulk speed (bottom) for different 3D VDF acquisition times. The values are normalized to their averaged input values over each time interval for a full 3D VDF measurement. The average plasma density is underestimated by less than $1 \%$, the average plasma temperature is underestimated by $\sim 2.5 \%$ and increases slightly with acquisition time, while the average plasma speed is practically calculated with no error. The measured moments exhibit an increasing standard deviation, shown as the red area, as the acquisition time increases. The standard deviation of the normalized density increases from $\sim 1 \%$ to $3.5 \%$ as the acquisition time increases from 1 to $\sim 100 \mathrm{~s}$. Within the same range of acquisition times, the standard deviation of the normalized plasma temperature increases from $<1 \%$ to $2 \%$. The standard deviation of the normalized derived speed is $<1 \%$ for the acquisition times we examine here. For comparison, we indicate the acquisition times of specific missions at the top of the plot.

temperature, and bulk speed as derived from the analysis of 200 observations sampled at random time intervals in our modeled turbulence. These are represented by the red histograms in Figure 3. Overlaid in each panel, we also show a histogram of the mean values of the corresponding input moments over each of the 200 observations (in black). These are the time averages of the input plasma moments, over each of the 200 full 3D instrument scans (approximately $1 \mathrm{~s}$ each). Besides small systematic errors associated with the numerical calculation of moments (see also the related discussion in the next section), the difference between the standard deviations of the derived and the input parameters, indicate that the error of the derived parameters due to turbulence is remarkably small. Note again that the statistical error of the derived plasma parameters presented here is due to turbulence only, as we do not include any other source of statistical error in our model. 

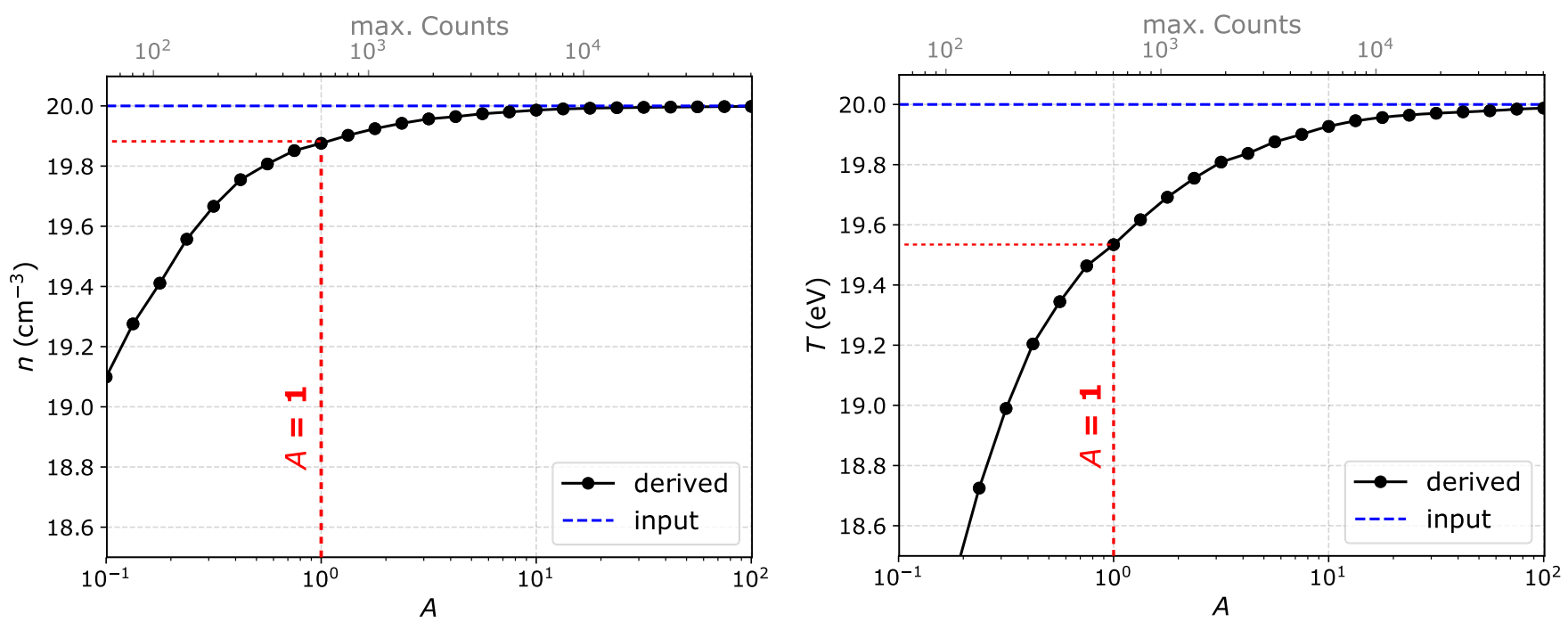

Figure 5. The derived (left) density and (right) temperature as functions of the instrument's efficiency expressed in terms of the efficiency multiplier $A$, for solar wind in the absence of turbulence with $n_{0}=20 \mathrm{~cm}^{-3}, T_{0}=20 \mathrm{eV}$, and $u_{0}=500 \mathrm{~km} \mathrm{~s}^{-1}$. The blue dashed lines indicate the values of the input parameters, and the red dashed indicate the efficiency we use in all of our model calculations for the turbulent solar wind and the corresponding derived parameters. If $A$ is small, the distribution function is not fully resolved, therefore the derived $n$ and $T$ are underestimated. The derived parameters approach asymptotically the actual plasma parameters as $A$ increases (see the text for more).

For comparison, we now study the effect of turbulence on measurements taken with different acquisition times. We specifically examine 3D VDF acquisition times ranging from 0.1 to $\sim 100 \mathrm{~s}$. For each acquisition time, we construct 200 modeled observations recorded at random time intervals in our model turbulence. We normalize the derived density, temperature, and bulk speed of each observation to their average values of the corresponding input moments within the specific acquisition. In Figure 4, we show the mean values (dots) and the standard deviations (red area) of the normalized derived plasma parameters as functions of the acquisition time. As the acquisition time increases, the uncertainty of the moments increases. The plasma density shows the greatest deviation, while the plasma speed shows the smallest deviation. In addition, the derived plasma temperature slightly increases with acquisition time, as expected from the analyses by Verscharen \& Marsch (2011) and Nicolaou et al. (2015a). Nevertheless, even for the highest acquisition time shown, the standard deviation of the derived parameters lies within a few percent of the corresponding average value. In the same figure, we also note the acquisition time of previous missions.

\section{Discussion and Conclusions}

Our analysis suggests that typical plasma fluctuations due to solar wind turbulence have only minor effects on upcoming SWA-PAS observations. Figure 2 demonstrates that the expected measured plasma density and temperature are slightly affected by a realistic turbulence spectrum, while the effects on the estimation of the bulk speed are negligible. The histograms of the derived plasma parameters in Figure 3 indicate a small deviation from the corresponding input parameters. Even though the the plasma temperature input is constant with time at $20 \mathrm{eV}$ in our model, because the Alfvén wave and slowmode models used are isothermal, the derived temperature has a standard deviation of $\sim 0.06 \mathrm{eV}$.

Our comparative study (Figure 4) shows that the plasma turbulence affects the accuracy of SWA-PAS less than it affects the accuracy of previous missions, measuring plasma protons at lower time resolution. The standard deviation of the normalized derived density is $\sim 1 \%$ for the acquisition time of Solar Orbiter, while it is $\sim 2 \%$ for the acquisition time of Helios, and $3.5 \%$ for the acquisition time of Wind. The standard deviation of the normalized derived temperature is $<1 \%$ for the acquisition time of Solar Orbiter, $\sim 1 \%$ for the acquisition time of Helios, and 2\% for the acquisition time of Wind. The standard deviation of the normalized derived speed is $<1 \%$ for the range of acquisition times we examine here.

Figures 2-4 show that the plasma density and temperature are slightly underestimated by $<1 \%$ and $\sim 2.5 \%$ respectively. Although we intentionally do not include any source of error in our model, calculating the moments of a distribution function by integrating it in discrete steps, introduces such systematic errors. This error occurs due to the instrument's finite and discrete angular and energy resolution (see also Figure 6). That error depends on the plasma parameters, which we will address and correct for in the future, when a complete error analysis of SWA-PAS is available.

In addition, due to limited efficiency, the instrument cannot resolve the full tails of the distribution function characterized by particle fluxes that are too low to produce detectable signal. As a result, the undetected particles do not contribute to the mathematical calculation of the moments, resulting in an underestimation of the plasma density and temperature (e.g., Nicolaou \& Livadiotis 2016; Nicolaou et al. 2018). We demonstrate this effect in Figure 5, where we plot the derived density and temperature as functions of the instrument efficiency, considering a non-fluctuating plasma with the same background parameters as our turbulent solar wind model (Section 3). We scale the model instrument's geometric factor $G$ by an efficiency multiplier $A$ (i.e., $G \rightarrow A G$ ) for each synthetic sample. For the value used in this work (i.e., $A=1$, red dashed in Figure 5) the error is similar to our results presented in Section 4. Moreover, the calculated $n$ and $T$ exhibit an asymptotic behavior, approaching the corresponding input values as $A$ increases. We note that in this work we consider constant (with energy and look direction) efficiency (see also Section 3.2), while the actual instrument efficiency 


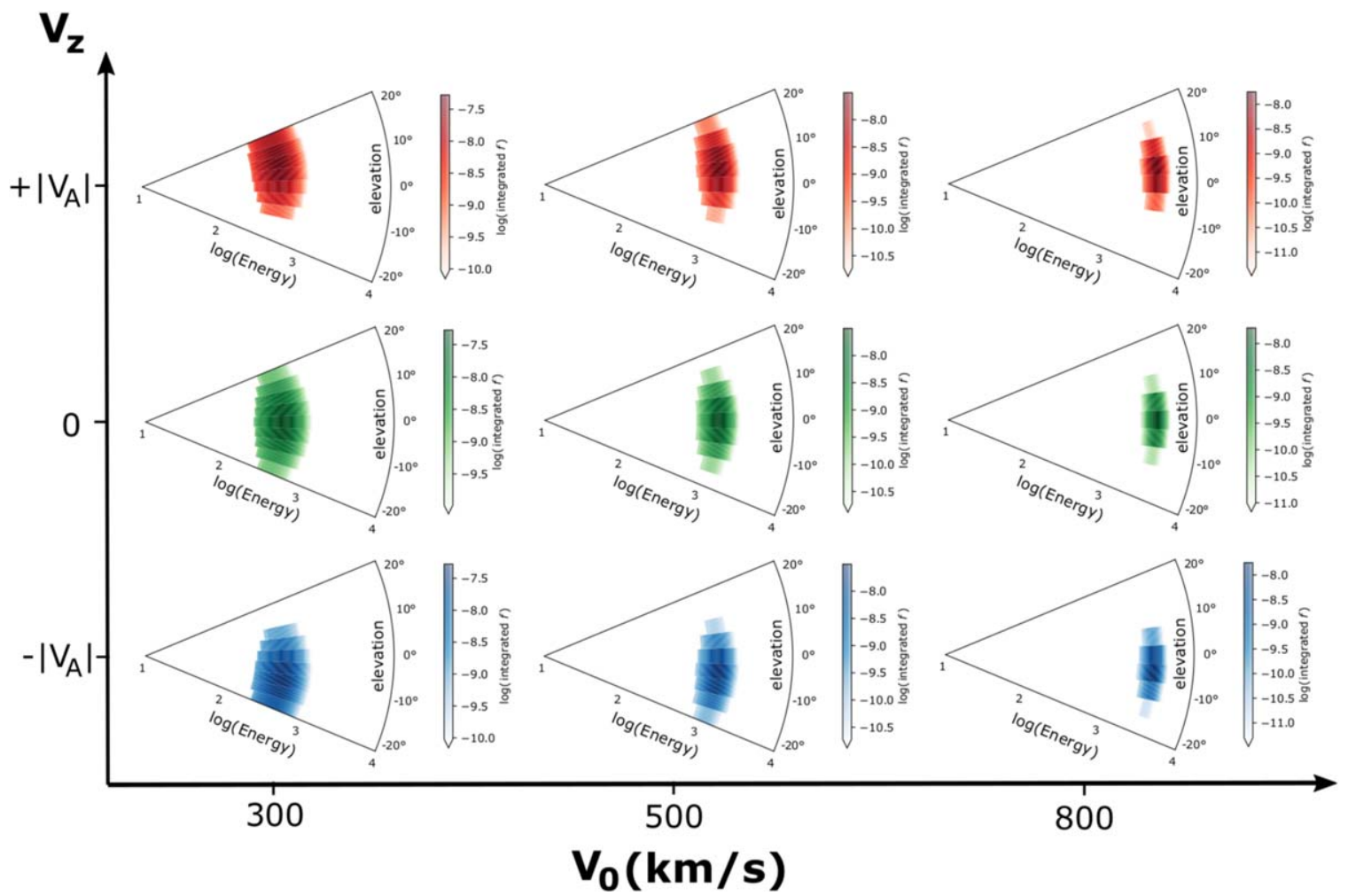

Figure 6. Plasma distribution functions in the instrument frame (integrated over azimuth direction). We assume a plasma with $n_{0}=20 \mathrm{~cm}^{-3}, T_{0}=20 \mathrm{eV}$ and three different background bulk velocities: (left) $u_{0}=300 \mathrm{~km} \mathrm{~s}^{-1}$, (middle) $500 \mathrm{~km} \mathrm{~s}^{-1}$, and (right) $800 \mathrm{~km} \mathrm{~s}^{-1}$. For all the examples, $V_{\mathrm{A}}=50 \mathrm{~km} \mathrm{~s}{ }^{-1}$ and for each $u_{0}$ we consider the following cases: (bottom) $\boldsymbol{u}=u_{0} \hat{x}-V_{\mathrm{A}} \hat{z}$, (middle) $\boldsymbol{u}=u_{0} \hat{x}$, and (top) $\boldsymbol{u}=u_{0} \hat{x}+V_{\mathrm{A}} \hat{z}$. The angular deviation of the center of the distribution increases with decreasing $u_{0}$, such that a significant portion of the distribution can leave the F.O.V. if $V_{\mathrm{A}} / u_{0}$ is large.

may vary by $40 \%$, as it is a complicated function of energy, azimuth, and elevation direction. As seen in Figure 5, efficiency variations by this amount can slightly affect the estimated moments by $\sim 1 \%$.

The VDF of a turbulent plasma is fluctuating in velocity space. On the other hand, the F.O.V. and energy range of the instrument are finite and cannot capture the entire velocity space. If the VDF is broader than or not entirely inside the instrument's F.O.V., the calculated moments are systematically underestimated. In Figure 6, for example, we show the distribution functions of a plasma with $n_{0}=20 \mathrm{~cm}^{-3}$, $T_{0}=20 \mathrm{eV}$ and three different background bulk velocities: $u_{0}=300,500$, and $800 \mathrm{~km} \mathrm{~s}^{-1}$, respectively. For simplicity we set $\boldsymbol{u}_{0}$ direction along the center of the F.O.V ( $x$-direction). We set $V_{\mathrm{A}} \sim 50 \mathrm{~km} \mathrm{~s}^{-1}$ and we let the bulk $u_{z}$ component to fluctuate between $-V_{\mathrm{A}}$ and $+V_{\mathrm{A}}$. As the ratio $V_{\mathrm{A}} / u_{0}$ increases, parts of the distribution function extend beyond the instrument's sampling range, causing an underestimation of the calculated moments. The underestimation of the moments is magnified as the distribution gets broader, which is the case for larger $T_{0} / u_{0}$. In addition, the instrument's absolute energy resolution decreases with energy, keeping $\Delta E / E=$ constant. Therefore, the instrument's ability to resolve the fluctuations decreases with increasing $u_{0}$. We conclude that the magnitude of the systematic errors varies with the plasma bulk parameters, but a detailed quantification of this effect is beyond the scope of this paper and the subject of a future study.

A detailed characterization of specific future data sets should adopt our methods by adjusting our turbulence model to the specific plasma conditions. We anticipate that the accuracy of the plasma moments depends on the plasma background parameters in a rather complicated way. Not only the amplitude and polarization properties of the fluctuations may change with changing plasma parameters in the solar wind, but also our instrument's ability to resolve them depends on the plasma parameters such as density, temperature, etc.

Furthermore, our turbulence model can be extended to include additional types of fluctuations such as fast-modes, whistler modes, and coherent and pressure-balanced structures as discussed by Lacombe et al. (2014), Klein et al. (2012), and Roberts et al. (2017). Such an extension would resemble the expected nature of the fluctuations more accurately; however, a detailed study of this type is beyond the scope of our work. Advanced modeling of the plasma observations could address additional sources of error that contribute to the total error in the derived plasma parameters. For instance, we note the contribution of the statistical counting error in any plasma measurements. According to counting statistics, every recorded number of particles $C$ has uncertainly $\delta C=\sqrt{C}$ (e.g., Wilson et al. 2008, 2012a, 2012b; Livi et al. 2014; Nicolaou et al. 2014, 2015a, 2015b, 2018; Wilson 2015; Elliott et al. 2016). The relative statistical error $1 / \sqrt{C}$ increases with decreasing counts, and could potentially propagate significant errors in the derived moments. As a rule of thumb, the statistical error increases with decreasing plasma flux through the instrument's aperture. Therefore, we expect larger statistical errors at larger heliocentric distances where the average plasma density is lower. A detailed characterization of the statistical error in SWA-PAS measurements is an ongoing project that we will combine with the findings of this paper in order to characterize the future observations. 
G.N. and C.J.O. are supported by the STFC Consolidated Grants to UCL/MSSL, ST/N000722/1 and ST/S000240/1. D.V. is supported by STFC Ernest Rutherford Fellowship ST/ 9003826/1. R.T.W. is supported by the STFC Consolidated Grant to UCL/MSSL, ST/S000240/1. The authors thank Andrey Fedorov, Ali Varsani, and Dhiren Kataria for helpful discussions.

\section{Appendix \\ Model of Turbulence Spectrum}

While the analysis in Section 4 is performed in the spacecraft frame $\left(x^{\prime}, y^{\prime}, z^{\prime}\right)$, we now adopt a coordinate system $(x, y, z)$ in which the background magnetic field $\boldsymbol{B}_{0}$ is parallel to $\hat{z}$. Both reference frames are connected through a rotation around the common $y^{\prime} / y$ axis. We define the background density $n_{0}$, background temperature $T_{0}$, and background velocity $\boldsymbol{u}_{0}$. We model plasma turbulence through a superposition of Alfvénic $\left(\Delta B_{\mathrm{A}}\right)$ and slow-mode $\left(\Delta B_{\mathrm{S}}\right)$ fluctuations:

$$
\Delta \boldsymbol{B}(t)=\Delta \boldsymbol{B}_{\mathrm{A}}(t)+\Delta \boldsymbol{B}_{\mathrm{S}}(t)=C_{1} \sum_{i} \delta \boldsymbol{B}_{i}(t)+C_{2} \sum_{j} \delta \boldsymbol{B}_{j}(t),
$$

where $C_{1}$ and $C_{2}$ are normalization constants.

Similarly, the plasma density fluctuations are

$$
\Delta n(t)=\Delta n_{\mathrm{A}}(t)+\Delta n_{\mathrm{S}}(t),
$$

and the components of the velocity fluctuations $\Delta \boldsymbol{u}(t)$ are

$$
\Delta u_{\|}(t)=\Delta u_{\| \mathrm{A}}(t)+\Delta u_{\| \mathrm{S}}(t)
$$

and

$$
\Delta \boldsymbol{u}_{\perp}(t)=\Delta \boldsymbol{u}_{\perp \mathrm{A}}(t)+\Delta \boldsymbol{u}_{\perp \mathrm{S}}(t) .
$$

The magnetic field and plasma fluctuations are convected over the spacecraft and thus only functions of time $t$. In our model, we consider the plasma particles to follow a Maxwell distribution function with changing bulk parameters:

$$
\begin{aligned}
f(\boldsymbol{u}, t)= & \left(n_{0}+\Delta n(t)\right)\left(\frac{m}{2 \pi k_{\mathrm{B}} T_{0}}\right)^{3 / 2} \\
& \times \exp \left(-\frac{m\left[\boldsymbol{u}-\left(\boldsymbol{u}_{0}+\Delta \boldsymbol{u}(t)\right)\right]^{2}}{2 k_{\mathrm{B}} T_{0}}\right),
\end{aligned}
$$

where $k_{\mathrm{B}}$ is the Boltzmann constant.

In the following subsections, we describe in detail the simulation setup for the Alfvén and slow-mode waves.

\section{A.1. Alfvén-wave Spectrum}

For each Alfvén wave harmonic, we assume

$$
\delta \boldsymbol{B}_{i}=\boldsymbol{A}_{i} \cos \left(\boldsymbol{k}_{i} \cdot \boldsymbol{u}_{0} t+\Psi_{i}\right),
$$

where $\boldsymbol{A}$ is the amplitude, $\boldsymbol{k}$ the wave vector, and $\Psi_{i}$ the phase. In Equation (11), we adopt Taylor's hypothesis, assuming that the observed fluctuations are due to the convection of frozen turbulence in the mean flow of the solar wind with velocity $\boldsymbol{u}_{0}$.

Each harmonic $\delta \boldsymbol{B}_{i}$ has a wave vector $\boldsymbol{k}$ with components:

$$
\begin{aligned}
& k_{x}=k \sin \theta_{k} \cos \phi_{k}, \\
& k_{y}=k \sin \theta_{k} \sin \phi_{k}, \\
& k_{z}=k \cos \theta_{k},
\end{aligned}
$$

where $\theta_{k}$ is the angle between $\boldsymbol{k}$ and $\boldsymbol{B}$ and $\phi_{k}$ the azimuthal angle of $\boldsymbol{k}$. We define the components of $\boldsymbol{k}$ along and perpendicular to $\boldsymbol{B}$ as $k_{\|}$and $k_{\perp}$, respectively. Then, according to Equation (12),

$$
\begin{aligned}
k_{\|} & =k \cos \theta_{k}, \\
k_{\perp} & =k \sin \theta_{k} .
\end{aligned}
$$

In our model, we consider the superposition of waves with a $k_{\perp}$ component with

$$
10^{-4} \leqslant k_{\perp} \rho_{\mathrm{g}}<10^{3},
$$

where $\rho_{\mathrm{g}}$ the proton gyroradius. We model fluctuations with $k_{\perp} \rho_{\mathrm{g}} \leqslant 1$ as Alfvén waves (AWs), and those with $k_{\perp} \rho_{\mathrm{g}}>1$ as kinetic Alfvén waves (kAWs). We discretize our spectrum in $71 k_{\perp}$ steps; 41 steps within the range of AWs and 30 within the range of kAWs. As in Chandran et al. (2010), for the amplitude of each harmonic, we set

$$
|\boldsymbol{A}|=10^{-4 / 3} B_{0}\left(k_{\perp} \rho_{\mathrm{g}}\right)^{-\gamma_{s}}
$$

where the spectral index

$$
\gamma_{s}=\left\{\begin{array}{lll}
1 / 3 & \text { for } & k_{\perp} \rho_{\mathrm{g}} \leqslant 1 \\
2 / 3 & \text { for } & k_{\perp} \rho_{\mathrm{g}}>1 .
\end{array}\right.
$$

The parallel component of the wave vector is

$$
k_{\|} \rho_{\mathrm{g}}=10^{-4 / 3}\left(k_{\perp} \rho_{\mathrm{g}}\right)^{\left(1-\gamma_{s}\right)},
$$

according to the critical-balance assumption (Goldreich \& Sridhar 1995). The spectrum is continuous at $k_{\perp} \rho_{\mathrm{g}}=1$, and $\boldsymbol{A} \perp \boldsymbol{B}_{0}$ and $\boldsymbol{A} \perp \boldsymbol{k}$. Equations (16) and (17) guarantee that, in the low-frequency limit, the turbulence is isotropic $\left(k_{\perp} \rho_{\mathrm{g}}=k_{\|} \rho_{\mathrm{g}}=10^{-4}\right)$ and becomes highly anisotropic with increasing frequency (e.g., Horbury et al. 2012; Chen 2016).

For each of the 71 values of $|\boldsymbol{k}|$, we set $101 \phi_{k}$ angle values, reaching from 0 to $2 \pi$ in uniform bins. In addition, for each combination of $|\boldsymbol{k}|$ and $\phi_{k}$, we include one wave propagating in the $+k_{\|}$-direction and one wave propagating in the $-k_{\|}$-direction. All $71 \times 101 \times 2=14,342$ waves that construct the spectrum have a different phase, randomly selected from the range from 0 to $2 \pi$. After some algebra, combining Equations (11) through (15), the sum in Equation (6) can be expressed as

$$
\begin{aligned}
\Delta B_{\mathrm{A}, x}= & C_{1} \sum_{l=1}^{71} \sum_{m=1}^{101} \sum_{n=1}^{2}-10^{-4 / 3} B_{0}\left(k_{\perp, l} \rho_{\mathrm{g}}\right)^{-\gamma_{s}} \sin \phi_{k, m} \\
& \times \cos \left[\left(k_{\perp, l} \cos \phi_{k, m} \boldsymbol{u}_{0} \cdot \hat{x}+k_{\perp, l} \sin \phi_{k, m} \boldsymbol{u}_{0} \cdot \hat{y}\right.\right. \\
& \left.\left.+(-1)^{n} k_{\|} \boldsymbol{u}_{0} \cdot \hat{z}\right) t+\Psi_{l m n}\right],
\end{aligned}
$$

and

$$
\begin{aligned}
\Delta B_{\mathrm{A}, y}= & C_{1} \sum_{l=1}^{71} \sum_{m=1}^{101} \sum_{n=1}^{2} 10^{-4 / 3} B_{0}\left(k_{\perp, l} \rho_{\mathrm{g}}\right)^{-\gamma_{s}} \cos \phi_{k, m} \\
& \times \cos \left[\left(k_{\perp, l} \cos \phi_{k, m} \boldsymbol{u}_{0} \cdot \hat{x}+k_{\perp, l} \sin \phi_{k, m} \boldsymbol{u}_{0} \cdot \hat{y}\right.\right. \\
& \left.\left.+(-1)^{n} k_{\|} \boldsymbol{u}_{0} \cdot \hat{z}\right) t+\Psi_{l m n}\right] .
\end{aligned}
$$

The density and velocity fluctuations are modeled as

$$
\frac{\Delta n_{\mathrm{A}}(t)}{n_{0}}=C_{1} \sum_{l=1}^{71} \sum_{m=1}^{101} \sum_{n=1}^{2} \xi_{l m n} \frac{\delta B_{\perp, l m n}(t)}{B_{0}},
$$



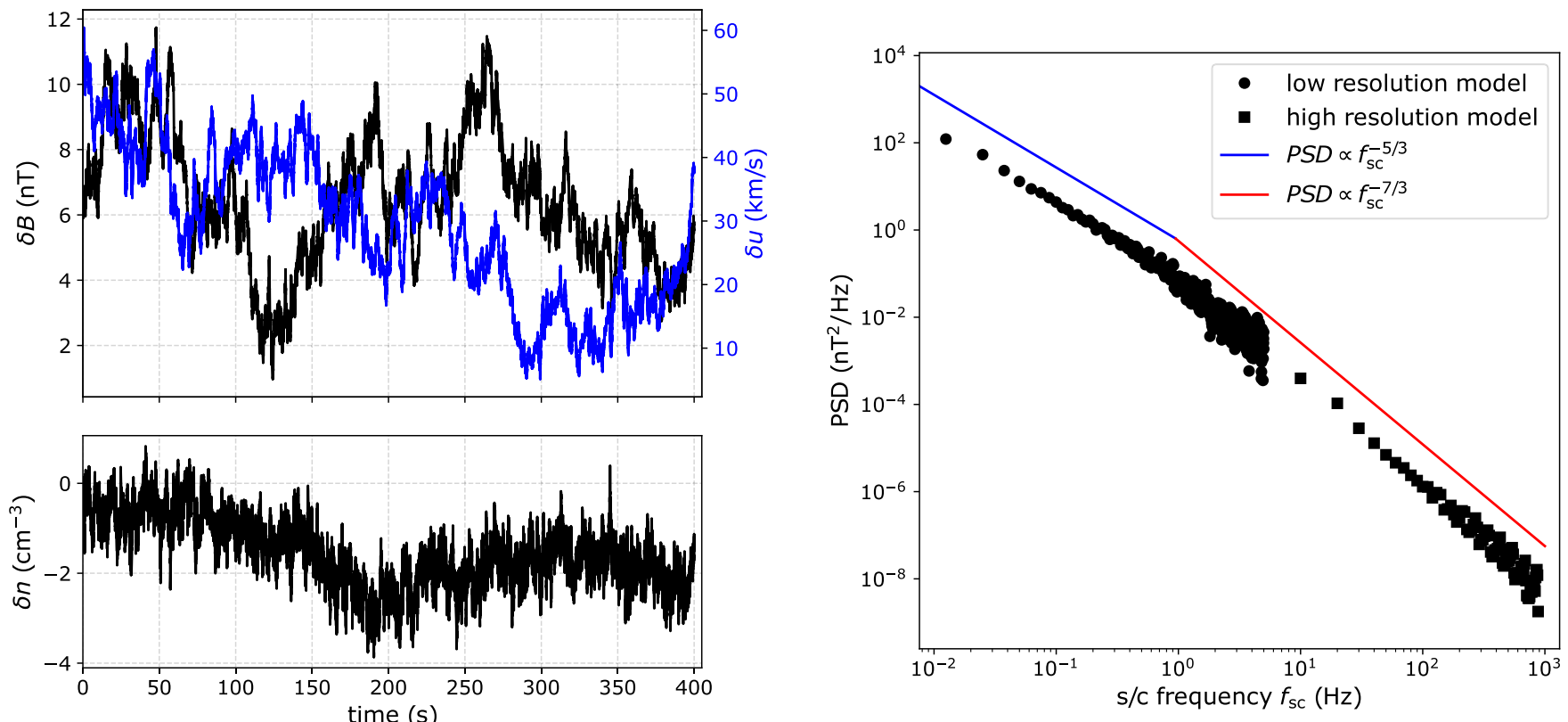

Figure 7. (Top left) Time series of the modeled magnetic field (black) and bulk speed (blue) fluctuations for a turbulent plasma in our model with background parameters $n_{0}=20 \mathrm{~cm}^{-3}, T_{0}=20 \mathrm{eV}, B_{0}=10 \mathrm{nT}$, and $u_{0}=500 \mathrm{~km} \mathrm{~s}^{-1}$. (Bottom left) Time series of the modeled density fluctuations. (Right) The power spectral density of magnetic-field fluctuations with combined low-resolution $\left(10^{-1} \mathrm{~s}\right.$, bullets) and high-resolution $\left(10^{-4} \mathrm{~s}\right.$, squares $)$ model data series. We overplot PSD $\propto f_{\mathrm{sc}}^{-5 / 3}$ (blue) and PSD $\propto f_{\mathrm{sc}}^{-7 / 3}$ (red) for reference.

$$
\frac{\Delta u_{\| \mathrm{A}}(t)}{V_{\mathrm{A}}}=C_{1} \sum_{l=1}^{71} \sum_{m=1}^{101} \sum_{n=1}^{2} \chi_{\|, l m n} \frac{\delta B_{\perp, l m n}(t)}{B_{0}},
$$

and

$$
\frac{\Delta \boldsymbol{u}_{\perp \mathrm{A}}(t)}{V_{\mathrm{A}}}=C_{1} \sum_{l=1}^{71} \sum_{m=1}^{101} \sum_{n=1}^{2} \chi_{\perp, l m n} \frac{\delta \boldsymbol{B}_{\perp, l m n}(t)}{B_{0}}
$$

for each harmonic $\delta \boldsymbol{B}$ of the spectrum, according to the twofluid solutions for $\xi_{l m n}, \chi_{\|, l m n}$, and $\chi_{\perp, l m n}$ by Hollweg (1999) and $\mathrm{Wu}$ et al. (2019). In Equations (21) and (22), $V_{\mathrm{A}}$ is the Alfvén speed.

The normalization constant $C_{1}$ is chosen so that the rootmean-square (rms) value of the magnetic-field fluctuations over a large interval $\Delta T$ is equal to the background magnetic field,

$$
C_{1}=\frac{B_{0}}{\sqrt{\frac{1}{\Delta T} \sum_{t=0}^{t=\Delta T}\left|\Delta \boldsymbol{B}_{A}(t)\right|^{2}}} .
$$

For Equation (23), we use $\Delta T \sim 10^{5} \mathrm{~s}$, which is $\sim 10$ times larger than the period of the isotropic fluctuations with $k_{\perp} \rho_{\mathrm{g}}=10^{-4}$.

\section{A.2. Slow-mode Spectrum}

The harmonics of the slow modes follow the same form as Equation (11). In our model, we simulate slow modes with

$$
10^{-4} \leqslant k_{\perp} \rho_{\mathrm{g}} \leqslant 1 .
$$

Equations (15) through (17) also apply to the slow modes in our model. The dimensionless factors $\xi_{l m n}$ and $\chi_{\|, l m n}$ are calculated with the same approach used by Verscharen et al. (2016, 2017). The normalization constant $C_{2}$ is chosen so that the total amplitude of compressive slow-mode-like fluctuations is $10 \%$ of the total amplitude of the incompressive Alfénic fluctuations:

$$
C_{2}=\frac{0.1 B_{0}}{\sqrt{\frac{1}{\Delta T} \sum_{t=0}^{t=\Delta T}\left|\Delta B_{S}(t)\right|^{2}}} .
$$

We model density and velocity fluctuations to construct the VDF using Equation (10) for $B_{0}=10 \mathrm{nT}, u_{0}=500 \mathrm{~km} \mathrm{~s}^{-1}$, $T_{0}=20 \mathrm{eV}$, and $n_{0}=20 \mathrm{~cm}^{-3}$. We set the bulk velocity along the spacecraft's $\hat{x}$ direction (anti-sunward along Sun-spacecraft line) and the magnetic-field vector $45^{\circ}$ elevated in the $x-y$ (top hat) plane. We model time series with a resolution $10^{-4} \mathrm{~s}$, which is 10 times shorter than the SWA-PAS acquisition time for one energy and one elevation direction. We also model time series with a lower resolution $\left(10^{-1} \mathrm{~s}\right)$, which we use only to examine the modeled spectrum in the lower frequency domain. The top left panel of Figure 7 shows a time series of $400 \mathrm{~s}$ of the high-resolution modeled magnetic field and proton speed fluctuations. The bottom left panel shows a time series of the density fluctuations for the same time interval, while the panel on the right shows the power spectral density of the magneticfield fluctuations, combining both the $10^{-4}$ and the $10^{-1} \mathrm{~s}$ resolution models. The spectral density follows the expected $f_{\mathrm{sc}}^{-5 / 3}$ and $f_{\mathrm{sc}}^{-7 / 3}$ profiles. Note that half of the harmonics in our spectrum propagate along the magnetic field, while the other half propagate in the opposite direction. Therefore, we do not observe any consistent correlation or anti-correlation between the magnetic field and the plasma fluctuations in Figure 7. Test studies of imbalanced turbulence using the extreme cases in which all waves propagate (i) parallel and (ii) anti-parallel to $\boldsymbol{B}_{0}$ (not shown here) lead to very similar observations to the ones shown for the balanced case in this work. Despite the fact that imbalanced turbulence exhibits persistent averaged correlations or anti-correlations between $\boldsymbol{B}$ and $\boldsymbol{u}$, the virtually identical spectra compared to the one we use (Figure 7) lead to almost identical results. 


\section{ORCID iDs}

G. Nicolaou (1) https://orcid.org/0000-0003-3623-4928

D. Verscharen (1) https://orcid.org/0000-0002-0497-1096

R. T. Wicks (1) https://orcid.org/0000-0002-0622-5302

C. J. Owen (1) https://orcid.org/0000-0002-5982-4667

\section{References}

Barouch, E. 1977, JGR, 82, 1493

Belcher, J. W., \& Davis, L. 1971, JGR, 76, 3534

Bourouaine, S., \& Perez, J. C. 2018, ApJL, 858, L20

Bruno, R., \& Carbone, V. 2013, LRSP, 10, 2

Cara, A., Lavraud, B., Fedorov, A., et al. 2017, JGR, 122, 1439

Chandran, B. D. G., Li, B., Rogers, B. N., Quataert, E., \& Germaschewski, K. 2010, ApJ, 720, 503

Chen, C. H. K. 2016, JPIPh, 82, 535820602

Elliott, H. A., McComas, D. J., Valek, P., et al. 2016, ApJS, 223, 19

Freeman, J. W. 1988, GeoRL, 15, 88

Gary, S. P., \& Smith, C. W. 2009, JGR, 114, A12105

Goldreich, P., \& Sridhar, S. 1995, ApJ, 438, 763

Goldstein, M. L., Roberts, D. A., \& Matthaeus, W. H. 1995, ARA\&A, 33, 283

Hollweg, J. V. 1999, JGR, 104, 14811

Horbury, T. S., Wicks, R. T., \& Chen, C. H. K. 2012, SSRv, 172, 325

Howes, G. G., Klein, K. G., \& TenBarge, J. M. 2014, ApJ, 789, 106

Kim, T. K., Ebert, R. W., Valek, P. W., et al. 2019, JGR, in press

Klein, K. G., Howes, G. G., TenBarge, J. M., et al. 2012, ApJ, 755, 159

Klein, K. G., Howes, G. G., \& TenBarge, J. M. 2014, ApJL, 790, L20

Klein, K. G., Perez, J. C., Verscharen, D., Mallet, A., \& Chandran, B. D. G. 2015, ApJL, 801, L18

Lacombe, C., Alexandrova, O., Matteini, L., et al. 2014, ApJ, 796, 5

Lin, R. P., Anderson, K. A., Ashford, S., et al. 1995, SSRv, 71, 125

Livi, R. J., Burch, J. L., Crary, F., et al. 2014, JGRA, 119, 3683

Marsch, E. 2006, LRSP, 3, 1

Matthaeus, W. H., Wan, M., Servidio, S., et al. 2015, RSPTA, 373, 20140154

McComas, D. J., Allegrini, F., Bagenal, F., et al. 2008, SSRv, 140, 261
Narita, Y. 2017, AnGeo, 35, 325

Nicolaou, G., \& Livadiotis, G. 2016, Ap\&SS, 361, 359

Nicolaou, G., Livadiotis, G., Owen, C. J., Verscharen, D., \& Wicks, R. T. 2018, ApJ, 864, 3

Nicolaou, G., McComas, D. J., Bagenal, F., \& Elliott, H. A. 2014, JGRA, 119,3463

Nicolaou, G., McComas, D. J., Bagenal, F., Elliott, H. A., \& Ebert, R. W. 2015a, P\&SS, 111, 116

Nicolaou, G., McComas, D. J., Bagenal, F., Elliott, H. A., \& Wilson, R. J. 2015b, P\&SS, 119, 222

Ogilvie, K. W., Chornay, D. J., Fritzenreiter, R. J., et al. 1995, SSRv, 71, 55

Perri, S., Servidio, S., Vaivads, A., \& Valentini, F. 2017, ApJS, 231, 4

Roberts, D. A. 2010, JGR, 115, A12101

Roberts, O. W., Narita, Y., Li, X., Escoubet, C. P., \& Laakso, H. 2017, JGRA, 122,6940

Rosenbauer, H., Schwenn, R., Marsch, E., et al. 1977, JGZG, 42, 561

Schwenn, R., Rosenbauer, H., \& Miggenrieder, H. 1975, RF, 19, 226

Taylor, G. I. 1938, RSPSA, 164, 476

Tu, C. Y., \& Marsch, E. 1995, SSRv, 73, 1

Vaivads, A., Retinò, A., Soucek, J., et al. 2016, JPIPh, 82, 905820501

Verscharen, D., Chandran, B. D. G., Klein, K. G., \& Quataert, E. 2016, ApJ, 831,128

Verscharen, D., Chen, C. H. K., \& Wicks, R. T. 2017, ApJ, 840, 106

Verscharen, D., Klein, K. G., \& Maruca, B. A. 2019, arXiv:1902.03448

Verscharen, D., \& Marsch, E. 2011, AnGeo, 29, 909

Wan, M., Matthaeus, W. H., Roytershteyn, V., et al. 2016, PhPl, 23, 042307

Wicks, R. T., Roberts, D. A., Mallet, A., et al. 2013, ApJ, 782, 118

Wilson, R. J. 2015, E\&SS, 2, 201

Wilson, R. J., Bagenal, F., Delamere, P. A., et al. 2013, JGRA, 118, 2122

Wilson, R. J., Bagenal, F., \& Persoon, A. M. 2017, JGRA, 122, 7256

Wilson, R. J., Crary, F., Gilbert, L. K., et al. 2012a, PDS Users Guide for Cassini Plasma Spectrometer (CAPS), Planetary Data System, http://ppi. pds.nasa.gov/

Wilson, R. J., Delamere, P. A., Bagenal, F., \& Masters, A. 2012b, JGR, 117, A 03212

Wilson, R. J., Tokar, R. L., Henderson, M. G., et al. 2008, JGR, 113, A12218

Wu, H., Verscharen, D., Wicks, R. T., et al. 2019, ApJ, 870, 106 